\title{
Carta al editor sobre el artículo «Frecuencia de diagnóstico de lesiones óseas en luxación glenohumeral anterior»
}

\author{
Comment to the article «Frecuency of diagnosis of bone lesions in cases of anterior glenohumeral dislocation» \\ Rivas-Ruiz R,* Hernández-Salinas LE ${ }^{\ddagger}$ \\ Instituto Mexicano del Seguro Social.
}

Comentarios al artículo «Frecuencia de diagnóstico de lesiones óseas en luxación glenohumeral anterior» escrito por el maestro Pablo Gustavo Galdámez-Grajeda y colaboradores.

El tema al cual hace referencia el M.C. Galdámez-Grajeda nos presenta el problema del subdiagnóstico de lesiones óseas producidas durante un evento de luxación glenohumeral, las cuales empobrecen el pronóstico funcional del paciente. ${ }^{1} \mathrm{El}$ artículo presentado refleja la necesidad de establecer un protocolo de actuación en los casos de luxaciones glenohumerales para realizar el diagnóstico oportuno de lesiones de la cabeza del húmero que, en caso de presentarse, se traducen en nuevos eventos de luxación; estos casos se presentan principalmente en pacientes menores de 40 años durante actividades deportivas y presentan eventos de reluxación en $47 \%$, asociado a lesiones producidas durante la luxación inicial, que condicionan inestabilidad subsecuente. ${ }^{2}$

Se desea comentar en cuanto al artículo que el diseño del protocolo se presenta claramente, mostrando el proceso seguido por los pacientes posterior a su captación para el ingreso al estudio, mediante el uso de flujogramas que presentan de forma didáctica los posibles caminos a seguir. Los esquemas, que abordan la forma correcta de realizar las proyecciones radiográficas especiales, permiten al personal médico y paramédico tener una imagen más clara y didáctica de la técnica para elaborar los estudios que la simple descripción de éstos.

En relación con la metodología y con el fin de mejorar estudios posteriores que tomen estos datos como base, debo hacer algunas aclaraciones sobre puntos metodológicos y estadísticos. El primero alude al diseño del estudio debido a que no se reporta la forma en la que se realizó el cálculo de tamaño de muestra, siendo este un paso importante para otorgar validez y precisión al estudio ${ }^{3}$ y debe ser aclarado.

En el apartado de resultados las variables se manejaron de forma inexacta. La variable «número de eventos previos de luxación» se presentó como una variable cuantitativa, cuando los eventos de luxación no se pueden dividir en fracciones de evento, por lo tanto, deberán ser tratadas como una variable ordinal, esto es relevante pues al presentar los resultados mediante el uso de media y desviación estándar nos aportan resultados equívocos, este tipo de variables deben presentarse como mediana y rangos intercuartilares y se analizan mediante el uso de pruebas no paramétricas. ${ }^{4}$

En referencia al «tiempo que permanece luxado», esta variable sí es cuantitativa, puesto que las unidades de tiempo pueden subdividirse y se presentan a lo largo de un continuo de datos, pero no presenta una distribución normal, esto se aprecia con la media y desviación estándar, al aplicar la fórmula $\bar{x} \pm 2 \mathrm{DE}$, se obtienen resultados sin factibilidad biológica; amplío esta explicación: una población se distribuye de forma normal por encima y debajo de su media, encontrando más allá de las dos desviaciones estándar solamente $2 \%$ de la población, tanto hacia los límites superiores como inferiores, encontrando por tanto dentro de estos límites de $\bar{x} \pm 2 \mathrm{DE}$ al $96 \%$ de la población total, por lo tanto, el resultado obtenido en la fórmula previamente comentada debe ser posible biológicamente hablando. Retomamos los datos obtenidos en esa variable $\bar{x}=95.11$, DE 53.328, sustituyamos en la fórmula $\bar{x} \pm 2 \mathrm{DE}: 95.11 \pm 2$ (53.328), obteniendo como resultado inferior -11.546 minutos que permanece luxado, no siendo posible este resultado.

\footnotetext{
* Doctor en ciencias, adscrito al Centro de Adiestramiento en Investigación Clínica, Coordinación de Investigación en Salud.

¥ Médico con Especialidad en Ortopedia, adscrito al Servicio de Urgencias, UMAE «Dr. Victorio de la Fuente Narváez».
}

Dirección para correspondencia: Dr. Luis Esteban Hernández-Salinas Tel: (55) 574735 00, ext. 25572

E-mail: luisestebanh@yahoo.com

https://dx.doi.org/10.35366/93353 doi: $10.35366 / 93353$

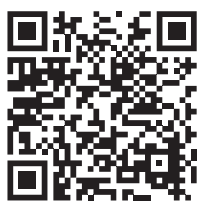


Estos métodos de análisis se conocen como métodos mentales, siendo el primero y de mayor peso la factibilidad biológica, que nos indica que los resultados deben ser biológicamente posibles, el segundo método de análisis consiste en las pruebas de normalidad de Kolmogorov y Shapiro, dependientes del número de participantes, donde obtendremos la asimetría y curtosis, como ya comentamos, la población debe distribuirse de forma simétrica a partir de su media, estas pruebas nos determinan si nuestra población en estudio está «cargada» hacia alguno de los lados (asimetría) o demasiado enfocada en la media (curtosis), indicándonos estas pruebas el manejo estadístico que deberá realizarse a la información obtenida.

Las variables en este estudio al presentarse mediante media y desviación estándar también fueron tratadas mediante pruebas paramétricas (t de Student), cuando se debieron utilizar pruebas no paramétricas (U de Mann-Whitney).

Estos errores en el procesamiento de la información nos brindan información incongruente y por tanto no reproducible. Discutir en nuestra revista estos problemas en la metodología y en el manejo de los datos nos ayudaría a los ortopedistas a mejorar la apreciación de las publicaciones de nuestra revista. Además, estamos seguros de que, al aclarar estos puntos, se obtendrá una mejoría en la metodología de los estudios subsecuentes, además de ser beneficioso para los lectores de la revista.

Referencias

1. Galdámez-Grajeda PG, Esperón-Hernández RI, Martínez-Escalante F, Bobadilla-Lezcano G, Cámara-Arrigunaga F. Frecuencia de diagnóstico de lesiones óseas en luxación glenohumeral anterior. Acta Ortopédica Mexicana. 2017; 31 (1): 12-7.

2. Kavaja L, Lähdeoja T, Malmivaara A, Paavola M. Treatment after traumatic shoulder dislocation: a systematic review with a network meta-analysis. Br J Sports Med. 2018; 52: 1498-506.

3. Talavera JO, Rivas-Ruiz R. Investigación clínica V. Tamaño de muestra. Rev Med Inst Mex Seguro Soc. 2011; 49 (5): 517-22.

4. Talavera JO, Rivas-Ruiz R. Investigación clínica IV. Pertinencia de la prueba estadística. Rev Med Inst Mex Seguro Soc. 2013; 49 (4): 401-5.

Esta carta al editor forma parte de la Iniciativa M, iniciativa que consiste en la mejoría de la calidad estadística de las publicaciones nacionales, así como utilizar como medio de difusión las revistas de origen nacional, ambas con el fin de incrementar la difusión de la ciencia entre profesionales médicos con habla hispana.

\title{
Respuesta a la carta al editor sobre el artículo: «Frecuencia de diagnóstico de lesiones óseas en luxación glenohumeral anterior».
}

\author{
Galdámez-Grajeda PG,* \\ Hospital de Ortopedia, Mérida, Yucatán, México
}

Estimado lector, ante todo agradecemos sus comentarios y nos permitimos aclarar las dudas que nos ha expresado al artículo «Frecuencia de diagnóstico de lesiones óseas en luxación glenohumeral anterior»: sobre la muestra, no se realizó alguna, se reportó a la población total $(\mathrm{N}=68)$ atendida durante el período referido. La Figura 4 ilustra el proceso de inclusión y distribución de los sujetos de estudio.

Con relación a las variables «número de eventos ...»y «número de intentos ...», efectivamente se manejaron como variables cuantitativas discretas, estamos de acuerdo que un sujeto no puede tener 1.5 luxaciones o intentos, pero en ese

* Médico Cirujano con Especialidad en Ortopedia y Traumatología, Fellow de Cirugía articular, Hospital de Ortopedia, Mérida, Yucatán, México.

Dirección para correspondencia:

Dr. Pablo Gustavo Galdámez-Grajeda

Km 16 Carretera

Interamericana, Terra Alta, Casa B31, Zona 1,

Mixco, CP 01057, Guatemala, CA.

E-mail: pablogaldamez@hotmail.com caso se presenta la media de casos e intentos por grupo de sujetos, con el propósito de conocer si existen o no diferencias entre ambos grupos, no la consideramos una variable ordinal dado que, como menciona Daniel, ${ }^{1}$ se asignan a los datos que se ordenan en categorías, no siendo el caso.

Respecto al uso de la prueba para la diferencia de medias «t de Student» ajustada como distribución t para muestras o grupos pequeños, si bien es deseable una distribución normal, la prueba puede tolerar desviaciones moderadas... siempre y cuando se obtenga una distribución en forma de montículo. ${ }^{1}$

Para finalizar no quisiera dejar pasar la oportunidad de agradecerle sus observaciones y comentarle que es estimulante y alentador el recibir opiniones sobre nuestro trabajo, estoy seguro que este es el camino adecuado para mejorar nuestra forma de análisis y presentación de nuestros resultados, por lo que tomaremos sus observaciones para publicaciones futuras.

Referencia

1. Daniel W. Bioestadística: base para el análisis de las ciencias de la salud. Edit. Limusa S.A. de C.V.; México, 2008. 6 y 163. 